\title{
Fenretinide targeting of human colon cancer sphere cells through cell cycle regulation and stress-responsive activities
}

\author{
LANLAN LIU ${ }^{1,2}$, JIANSHENG LIU ${ }^{3,4}$, HAIWEI WANG ${ }^{1}$, HUI ZHAO ${ }^{5}$ and YANZHI DU ${ }^{3,4}$ \\ ${ }^{1}$ Institute of Health Sciences, Shanghai Jiao Tong University School of Medicine (SJTUSM)/Shanghai Institutes for \\ Biological Sciences, Chinese Academy of Sciences, Shanghai 200025; ${ }^{2}$ Graduate School of The Chinese Academy of Sciences, \\ Shanghai 200031; ${ }^{3}$ Center for Reproductive Medicine, Ren Ji Hospital, School of Medicine, Shanghai Jiao Tong University; \\ ${ }^{4}$ Shanghai Key Laboratory for Assisted Reproduction and Reproductive Genetics, Shanghai 200135; \\ ${ }^{5}$ School of Biomedical Sciences, Faculty of Medicine, The Chinese University of Hong Kong, \\ Hong Kong 999077, SAR, P.R. China
}

Received September 26, 2015; Accepted March 10, 2017

DOI: $10.3892 / \mathrm{ol} .2018 .9296$

\begin{abstract}
Cancer stem cells (CSCs) are considered to be the main cause of chemoresistance and the resultant low survival rate of patients with cancer. $\mathrm{N}$-(4-hydroxyphenyl)-retinamide, known as fenretinide or 4HPR, is a synthetic derivative of all-trans-retinoic acid. It is a promising anticancer agent, has minimal side effects and synergizes with other anticancer agents to reinforce their anticancer efficacy. The present study investigated whether fenretinide eliminated colon sphere cells. HT29 and HCT116 cells incubated in low-serum culture medium were more sensitive to fenretinide treatment than those incubated in full-serum medium. Colon spheres formed in serum-free medium demonstrated stem-like characteristics. The percentage of cluster of differentiation (CD) $44^{+}$cells was significantly higher in sphere cells compared with parental cells. Sphere cells also demonstrated increased tumorigenic ability in non-obese diabetic/severe combined immunodeficiency mice. Fenretinide inhibited the formation of colon spheres in HT29 and HCT116 cells. Microarray, cell cycle and reverse transcription-quantitative polymerase chain reaction analysis revealed that fenretinide induced genes associated with cell cycle regulation and the stress response in fenretinide-treated HT29 sphere cells. To the best of our knowledge, the present study was the first to investigate the effect of fenretinide on colon stem cells. Fenretinide was demonstrated to preferentially target colon sphere cells, which may possess certain stem-like characteristics. These results are an important addition to the current knowledge concerning
\end{abstract}

Correspondence to: Professor Yanzhi Du, Center for Reproductive Medicine, Ren Ji Hospital, School of Medicine, Shanghai Jiao Tong University, 845 Lingshan Road, Shanghai 200135, P.R. China E-mail: yanzhidu@hotmail.com

Key words: cancer stem cells, sphere, colon cancer, fenretinide, cell cycle, cell stress fenretinide, and provide a foundation for its clinical application in the treatment of cancer.

\section{Introduction}

Despite continued research to identify novel antineoplastic agents, colon carcinoma remains the third most common neoplastic malignancy globally and one of the leading causes of cancer-associated mortality, due to its aggressiveness and resistance to therapy (1). The majority of patients respond to the initial treatment and achieve clinical remission following chemotherapy, but a substantial proportion of patients develop chemoresistance and relapse within 5 years, leading to a low survival rate. Chemoresistance and the resultant low survival rate are thought to be associated with the presence of cancer stem cells (CSCs), which represent a rare population of undifferentiated cells that drive the growth of tumors, differentiating into a variety of cell types corresponding to the original tissue while maintaining the ability for self-renewal (2).

Colon cancer stem-like cells were identified in 2007 (3). Another characteristic of CSCs is their relative quiescence, which may endow CSCs with resistance to well-defined chemotherapies that predominantly target proliferating rather than quiescent cells. Resistance is also exhibited through a wide spectrum of activities, including DNA damage repair, altered cell cycle checkpoint control, and malfunction of apoptosis, drug transporters and detoxifying enzymes (4). Therefore, one strategy for the treatment of chemo-resistant cancers is to develop an agent that selectively targets quiescent and drug-resistant CSCs (5).

Retinoids are a class of natural and synthetic derivatives of vitamin A that exert effects on critical biological processes that include development, cell growth and differentiation, metabolism and homeostasis. They demonstrate anti-proliferative and cell differentiation activity in various cancer cell lines in vitro and in vivo, and are therefore promising candidates for the chemoprevention and treatment of certain types of cancer $(6,7)$. However, their high degree of toxicity, which may reflect the broad biological responses mediated by retinoid receptors, is the main limitation for the clinical use of the 
retinoids available at present. $\mathrm{N}$-(4-hydroxyphenyl)-retinamide (fenretinide, also known as 4HPR), a synthetic derivative of all-trans-retinoic acid, has emerged as a promising anticancer agent due to its distinct advantages over other agents for treating cancer, as demonstrated in numerous in vitro and in vivo studies, and chemoprevention clinical trials (8-10). In addition to its efficacy against a wide range of types of tumor, fenretinide has minimal side effects and synergizes with other anticancer agents, reinforcing their anticancer efficacy (11-13).

In the present study, sphere culture in serum-free medium was used to isolate tumor spheres from two human colon cell lines: HT29 and HCT116. The capacity for self-renewal, chemoresistance, and tumor initiation was then assessed in the tumor sphere cells. Fenretinide was demonstrated to preferentially target colon sphere cells, which are believed to possess certain stem-like characteristics. Transcriptome analysis of fenretinide-treated HT29 sphere cells was then performed to investigate the mechanisms involved, and a number of features associated with cell cycle regulation and activation of reactive oxygen species (ROS)-induced stress responses were identified. These results are an important addition to the current knowledge concerning of fenretinide, and provide a foundation for its clinical application in the treatment of cancer.

\section{Materials and methods}

Cell lines, cell culture and reagent. The human colon cancer cell lines HCT116 and HT29, obtained from the Cell Bank of Type Culture Collection of the Chinese Academy of Sciences (Shanghai, China), were cultured in RPMI-1640 medium (Gibco; Thermo Fisher Scientific, Inc., Waltham, MA, USA) containing $10 \%$ fetal bovine serum (FBS; PAA Laboratories; GE Healthcare Life Sciences, Chalfont, UK). The sphere cells were obtained with similar protocol as illustrated in previous study (14). Single HCT116 and HT29 cells were plated in ultralow-attachment plates in serum-free RPMI-1640 medium at a density of 5,000 cells $/ \mathrm{ml}$. The sphere-forming medium (SFM) was Dulbecco's modified Eagle's medium-F12 (Gibco; Thermo Fisher Scientific, Inc.) supplemented with 2\% B-27, $20 \mathrm{ng} / \mathrm{ml}$ epidermal growth factor (Sigma-Aldrich; Merck KGaA, Darmstadt, Germany), $5 \mu \mathrm{g} / \mathrm{ml}$ insulin (Sigma-Aldrich; Merck KGaA) and 0.4\% FBS (Ameresco, Inc., Framingham, MA, USA). Dissociated cells were seeded in SFM with or without fenretinide treatment, and the spheres were observed and photographed with an objective lens at magnification, x20 using an inverted microscope. All cells were incubated at $37^{\circ} \mathrm{C}$ in a humidified atmosphere with $5 \% \mathrm{CO}_{2}$. Fenretinide was purchased from Sigma-Aldrich (Merck KGaA) and dissolved in absolute ethanol.

Cell cycle and cell viability assay. For the cell viability assay, HT29 and HCT116 cells were incubated at $37^{\circ} \mathrm{C}$ in 48 -well plates at a density of 10,000 cells/well overnight in RPMI-1640 medium containing 10 or $0.5 \%$ FBS when comparing the sensitivity of colon cancer cells to fenretinide in normal or low serum levels, respectively, and were then treated with $6 \mu \mathrm{M}$ fenretinide for 48 or 72 h. Fenretinide in absolute ethanol was used as the negative control. For cell cycle analysis, the trypsinized adherent cells were cultured for $48 \mathrm{~h}$, then collected and fixed with $75 \%$ ethanol (v/v) for $24 \mathrm{~h}$ at $4^{\circ} \mathrm{C}$, stained with propidium iodide at the final concentration of $50 \mu \mathrm{g} / \mathrm{ml}$ for $30 \mathrm{~min}$ at room temperature and analyzed by flow cytometry using the FC500 flow cytometer (Beckman Coulter, Inc., Brea, CA, USA). HT29 and HCT116 cells were treated with the MTT solution (50 $\mu \mathrm{l}$; $5 \mu \mathrm{g} / \mathrm{ml}$ in PBS) to each well and the plate was incubated for $3 \mathrm{~h}$ at $37^{\circ} \mathrm{C}$, following which the medium was replaced by $200 \mu \mathrm{l}$ dimethyl sulfoxide. Cell viability was evaluated by measuring the absorbance optical density at $595 \mathrm{~nm}$ on a DU $800 \mathrm{spec}-$ troscopy microplate reader (Beckman Coulter, Inc., Brea, CA, USA). For sphere cell viability, the cells were cultured in SFM for 7 days as described in the cell culture methods. The sphere cells were then centrifuged at $600 \mathrm{x}$ g for $5 \mathrm{~min}$ at room temperature and collected in a new $50 \mathrm{ml}$ centrifuge tube, then $0.05 \%$ trypsin (Gibco; Thermo Fisher Scientific, Inc., Waltham, MA, USA) was added for enzymatic dissociation. The sphere cells were incubated for $3 \mathrm{~min}$ at $37^{\circ} \mathrm{C}$, seeded in SFM in 48-well plates with 10,000 cells/well, and then treated with $20 \mu \mathrm{M}$ fluorouracil (5-FU; Sigma-Aldrich; Merck KGaA) or $400 \mathrm{ng} / \mathrm{ml}$ epirubicin (EPB; Sigma-Aldrich; Merck KGaA) at $37^{\circ} \mathrm{C}$ for $48 \mathrm{~h}$. Concurrently, the parental HT29 cells were incubated in 48 -well plates with 10,000 cells/well at $37^{\circ} \mathrm{C}$ overnight in RPMI-1640 medium containing $10 \% \mathrm{FBS}$, and were then treated with $20 \mu \mathrm{M} 5-\mathrm{FU}$ or $400 \mathrm{ng} / \mathrm{ml} \mathrm{EPB}$ for $48 \mathrm{~h}$ at $37^{\circ} \mathrm{C}$. Sterile normal saline was added into the plate as the negative control.

Cell apoptosis assay. For parental cell apoptosis assay, HT29 and HCT116 cells were seeded into 6 -well plates (2x10 $/$ well) and exposed to the fenretinide treatments with indicated concentration for indicated time. Absolute ethanol was used as the negative control. The treated cells were dissociated with $0.25 \%$ trypsin for $3 \mathrm{~min}$ at $37^{\circ} \mathrm{C}$, collected in BD falcon centrifuge tubes, washed with Annexin $\mathrm{V}$ binding buffer (BD Biosciences, San Jose, CA, USA) and centrifuged at $350 \mathrm{x}$ g for $5 \mathrm{~min}$. Washing and centrifugation was repeated twice. Then, the samples were incubated with $5 \mu$ l fluorescein isothiocyanate (FITC)-stained Annexin V antibody and $5 \mu \mathrm{l}$ propidium iodide stain for $15 \mathrm{~min}$ at room temperature according to the protocol of the manufacturer of the Apoptosis Detection Kit (BD Biosciences; cat. no. 556547), and detected and analyzed by flow cytometry using the FC500 flow cytometer (Beckman Coulter, Inc., Brea, CA, USA). For the sphere cell apoptosis assay, the sphere cells from HCT116 and HT29 were cultured in SFM, trypsinized, and seeded into 6-well plates with $2 \times 10^{5}$ cells/well. Following treatment with $3 \mu \mathrm{m}$ fenretinide, sphere cells were collected and centrifuged with $1,000 \mathrm{x} \mathrm{g}$ for $5 \mathrm{~min}$ at room temperature. Cell apoptosis was detected using a Fluorescein Isothiocyanate (FITC)-Annexin V Apoptosis Detection kit (BD Biosciences) according to the protocol of the manufacturer, and analyzed by flow cytometry. The total percentage of Annexin $\mathrm{V}^{+} / \mathrm{PI}^{-/+}$cells was quantified and CXP Analysis software (version 1.0; Beckman Coulter, Inc., Brea, CA, USA) was used to analyze the apoptosis data.

Cell surface marker analysis. HT29 parental cells at $80 \%$ confluence were rinsed twice with PBS, released from the culture dish with $1 \mathrm{mmol} / 1$ EDTA (Sigma-Aldrich; Merck $\mathrm{KGaA}$ ) for $10 \mathrm{~min}$ followed by $0.05 \%$ trypsin (Sigma-Aldrich; Merck KGaA) for $1 \mathrm{~min}$, washed with RPMI-1640 containing $10 \% \mathrm{FBS}$, and harvested by centrifugation at $300 \mathrm{x} \mathrm{g}$ for 
Table I. Forward and reverse primers of genes in reverse transcription quantitative polymerase chain reaction analysis.

\begin{tabular}{llc}
\hline Gene name & \multicolumn{1}{c}{ Forward primers } & Reverse primers \\
\hline CDC25A & GTTAGACGTCCTCCGTCCAT & AGACCTTTCCTTCCCAGGTT \\
CCNE2 & GCATTATGACACCACCGAAG & ATTGGCTAGGGCAATCAATC \\
E2F8 & CCGCAACAGAGATCAGAAAA & AAGTTCCTCTGCCACTTCGT \\
BRCA2 & AGTGACCTTCCAGGGACAAC & GCCCATTGATGGCTAAAACT \\
CCNA2 & GGAGCTGCCTTTCATTTAGC & TTGACTGTTGTGCATGCTGT \\
SESN2 & GAGGACTTCACTCGGAGAGG & GCATGGCGATGGTATTGTAG \\
TGM2 & CCAGAACAGCAACCTTCTCA & TCGTACTTGGTGCTCAGGTC \\
HERPUD1 & TGGATTGGACCTATTCAGCA & CAGGAGGAGGACCATCATTT \\
CLGN & AACCAATGGACCTGGAAGAG & CTCCAGATCCTGTGCTCTCA
\end{tabular}

CDC25A, cell division cycle 25A; CCNE2, cyclin E2; E2F8, E2F transcription factor 8; BRCA2, BRCA2, DNA repair associated; CCNA2, cyclin A2; SESN2, sestrin 2; TGM2, transglutaminase 2; HERPUD1, homocysteine inducible ER protein with ubiquitin like domain 1; CLGN, calmegin.

3 min at room temperature. Sphere cells from HT29 was cultured in SFM and obtained as described above. All cells were resuspended at $1 \times 10^{6}$ cells $/ \mathrm{ml}$ in cold PBS buffer with $1 \%$ FBS (Ameresco, Inc.) for $5 \mathrm{~min}$ at room temperature and then stained with FITC-conjugated cluster of differentiation CD44-specific antibodies (BD Biosciences; cat. no. 560977) at a dilution of 1:400 for $30 \mathrm{~min}$ in the dark at room temperature. For investigating the difference prior and subsequent to fenretinide treatment for sphere-derived HT29 cells, sphere cells derived from HT29 cells were seeded into 6-well at a density of $2 \times 10^{5}$ cells/well. Sphere cells were treated with $3 \mu \mathrm{M}$ fenretinide or absolute ethanol for $48 \mathrm{~h}$. Cells were harvested, resuspended, and stained with CD44-specific antibody as described above. The cells were analyzed by flow cytometry using FC500 flow cytometer (Beckman Coulter, Inc.). The data were analyzed with CXP analysis software (version 1.0; Beckman Coulter, Inc.).

Subcutaneous model of tumorigenesis. The animal experiments were approved by the Committee on Laboratory Animal Research of Shanghai Jiao Tong University (Shanghai, China), and were conducted according to the guidelines of the Laboratory Animal Center of Shanghai Jiao Tong University School of Medicine (Shanghai, China). Female non-obese diabetic/severe combined immunodeficiency (NOD/SCID) mice (6-8 weeks old, $25 \mathrm{~g}$ ) were purchased from Shanghai SLAC Animal Center (Shanghai, China) and fed ad libitum in a sterile environment and a relative humidity of $40-70 \%$ at $25^{\circ} \mathrm{C}$ in a $12 \mathrm{~h}$ light/dark cycle. A total of four mice were used to compare tumorigenic ability between HT29 sphere cells and parental cells. HT29 sphere cells $\left(1 \times 10^{4}\right.$ cells) were injected subcutaneously into the left inguinal area of each mouse and the same number of parental cells was injected into the right inguinal area of the same mouse. Tumor growth was monitored every 5 days, and the xenograft mice were sacrificed after 3 weeks when the tumor diameter of xenograft mice was $>15 \mathrm{~mm}$.

Reverse transcription-quantitative polymerase chain reaction $(R T-q P C R)$ analysis. Cellular RNA was isolated using TRIzol $^{\circledR}$ reagent (Molecular Research Center, Inc., Cincinnati,
$\mathrm{OH}$, USA) according to the manufacturer's protocol. DNA was removed from the samples using DNase treatment (DNA-free kit; Ambion; Thermo Fisher Scientific, Inc.), and cDNA was synthesized according to the Moloney Murine Leukemia Virus Reverse Transcription kit (Promega Corporation, Madison, WI, USA). The sequence of the GAPDH primer was as follows: forward, GCACCGTCAAGGCTGAGAAC; reverse, TGGTGAAGACGCCAGTGGA, and these sets were used to produce a normalization control. qPCR was performed in triplicate with SYBR-Green PCR Master Mix (Applied Biosystems; Thermo Fisher Scientific, Inc.) and the 7900HT Fast Real-Time PCR machine (Applied Biosystems; Thermo Fisher Scientific, Inc.) with the following thermocycling conditions: $95^{\circ} \mathrm{C}$ for $10 \mathrm{~min}$, followed by 40 cycles of $95^{\circ} \mathrm{C}$ for $15 \mathrm{sec}$ and $60^{\circ} \mathrm{C}$ for $1 \mathrm{~min}$, and a final elongation step of $72^{\circ} \mathrm{C}$ for $10 \mathrm{~min}$, was used to perform the reaction. The expression levels of certain cell-cycle and stress-response associated genes, including cyclin E2 (CCNE2), cell division cycle $25 \mathrm{~A}$ $(C D C 25 A), \mathrm{E} 2 \mathrm{~F}$ transcription factor $8(E 2 F 8)$, BRCA2, DNA repair associated $(B R C A 2)$, cyclin A2 (CCNA2), sestrin 2 (SESN2), transglutaminase 2 (TGM2), homocysteine inducible ER protein with ubiquitin like domain 1 (HERPUDl) and calmegin $(C L G N)$ were assessed. $G A P D H$ was used as a reference, and the $2^{-\Delta \Delta \mathrm{Cq}}$ method was used to quantify the relative expression level of those genes (15). Table I summarizes the forward primers and reverse primers of all other genes.

Microarray hybridization and data mining. Total RNA from HT29 parental and sphere cells was amplified and labeled with biotin according to the standard Affymetrix protocol as outlined by Lockhart et al (16). The fragmented, biotinylated cDNA was then subjected to hybridization with the GeneChip Human Genome-U133 Plus 2.0 array (Affymetrix, Inc., Santa Clara, CA, USA) according to manufacturer's protocol. Analysis of the underlying biological mechanisms of the significantly differentially expressed genes was performed using the Database for Annotation, Visualization and Integrated Discovery (DAVID 6.7 version) Bioinformatics Resources database (17), with functional relevance assessed via Gene Ontology (GO) enrichment analysis (18). Genes 

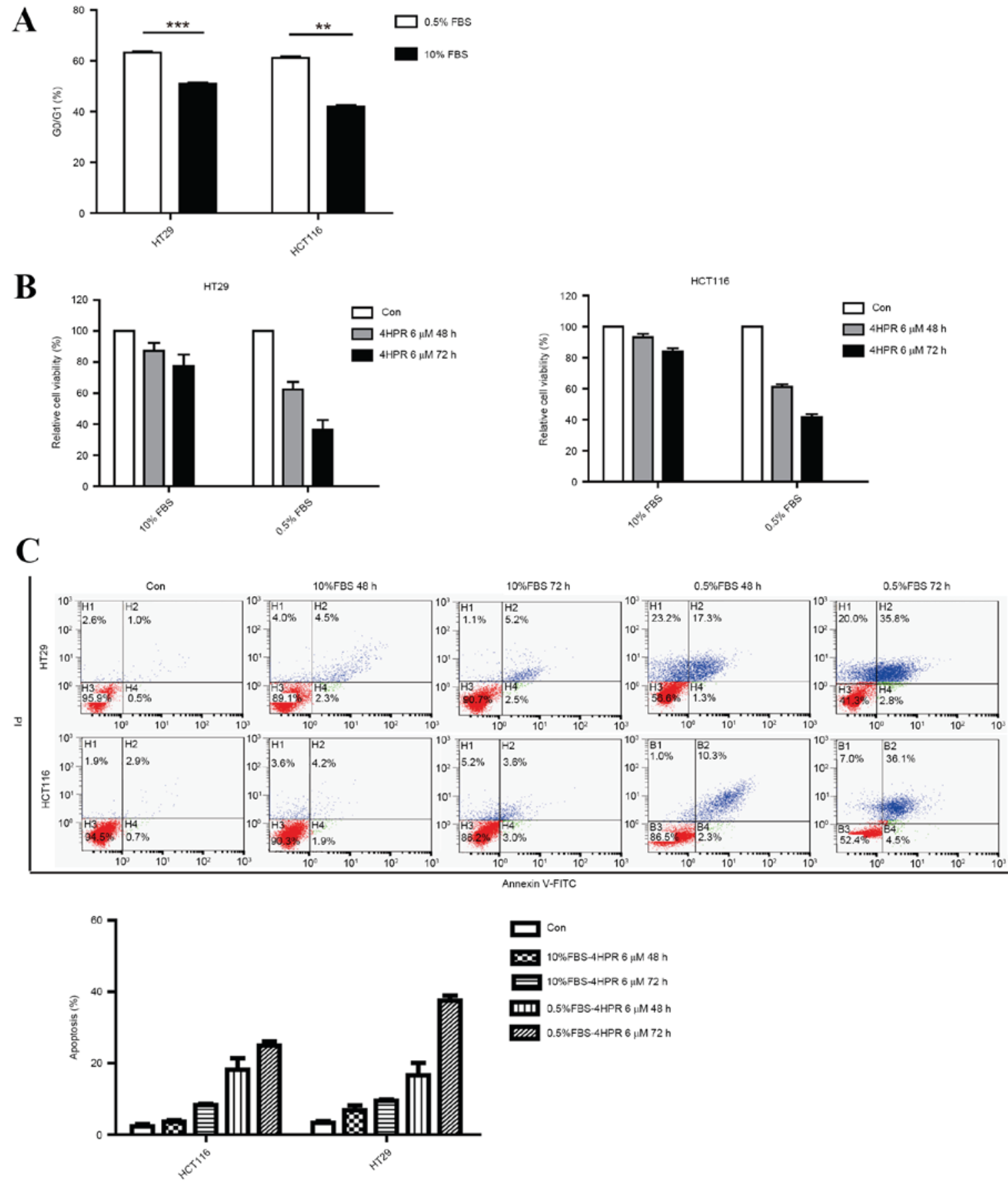

Figure 1. Cells cultured in low-serum medium displayed greater sensitivity to 4HPR. (A) Cells grown in low-serum medium were arrested in the G0 phase, with higher ratio of cells in $\mathrm{G}_{0} / \mathrm{G}_{1}$. (B) Viability of HT29 and HCT116 cell lines grown in normal or low-serum medium 48 or $72 \mathrm{~h}$ following treatment with 4HPR $(6 \mu \mathrm{M})$, relative to the negative control, as assessed by MTT assay. (C) HT29 and HCT116 cells were cultured in normal or low-serum medium were treated with $6 \mu \mathrm{M} 4 \mathrm{HPR}$ for 48 or $72 \mathrm{~h}$, and the proportion of apoptotic cells was assessed using an Annexin V/FITC assay. Data are expressed as the mean \pm standard error of the mean and are representative of at least three independent experiments. ${ }^{* *} \mathrm{P}<0.01$ and ${ }^{* * * *} \mathrm{P}<0.001$ vs. $0.5 \%$ FBS. 4HPR, fenretinide; FITC, fluorescein isothiocyante; FBS, fetal bovine serum.

that varied by $>2$-fold following fenretinide treatment in each sample were selected as the target genes, and mapped as Venn diagrams using VennyDiagram software (http://bioinfogp. cnb.csic.es/tools/venny/). The transcriptome profiles of HT29 enriched sphere cells treated with $6 \mu \mathrm{m}$ fenretinide for 48 and $72 \mathrm{~h}$ and the unenriched cells treated with $3 \mu \mathrm{m}$ fenretinide for 24 and $48 \mathrm{~h}$ were deposited in NCBI's Gene Expression Omnibus (GEO) and the microarray data are available at the GEO accession no. GSE66983 (19). The similar concentration of fenretinide and treatment time are described in our previous study (14). For data mining, a software package of Component Plane Presentation, Self-Organizing Map was implemented using Matlab 6.5 as described previously $(20,21)$.

Statistical analysis. Data and photographs were analyzed and drawn with GraphPad Prism v5 (GraphPad Software, Inc., La Jolla, CA, USA). All data were expressed as the mean \pm standard error of the mean. Statistical analysis was 
A

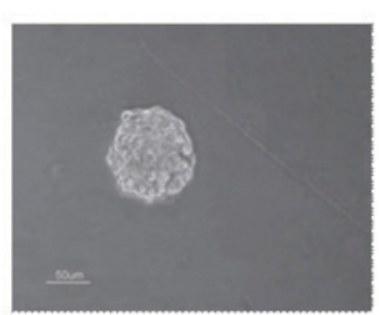

C

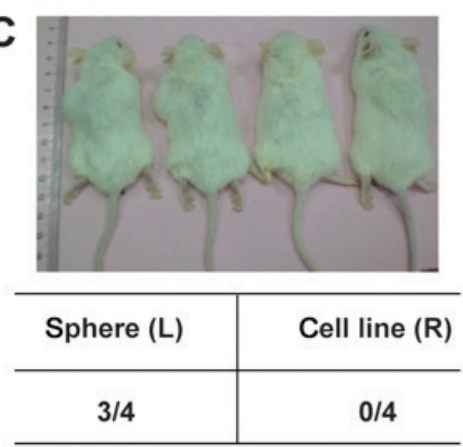

B
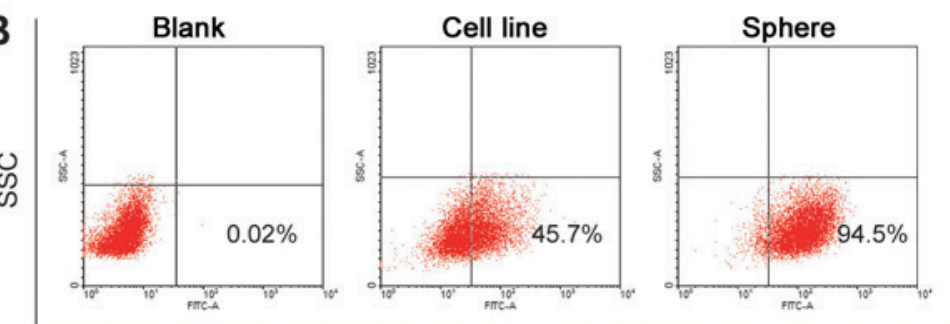

CD44-FITC

D

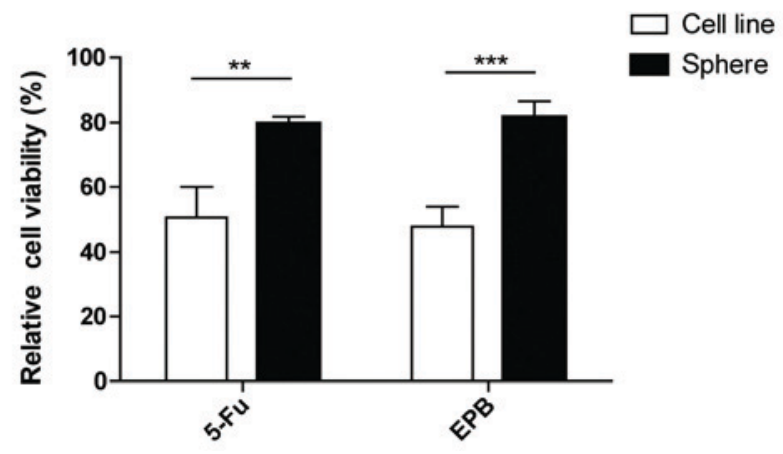

Figure 2. Spheres from HT29 and HCT116 cells exhibited stem cell-like characteristics in serum-free medium. (A) HT29 and HCT116 cells formed spheres under sphere-forming conditions. The sphere was photographed using an inverted microscope following the culture of a single cell in a 96-well suspension culture plate for 5 days. (B) Representative flow cytometry plots revealing the percentages of cluster of differentiation $44^{+}$cells in HT29 parental cells and sphere cells. (C) Comparison of the tumorigenic ability of HT29 parental cells and sphere cells in immune-deficient mice. (D) Comparison of the relative cell viability of the parental HT29 cells and the sphere cells treated with 5-FU or EPB. Data are expressed as the mean \pm standard error of the mean and are representative of three independent experiments. ${ }^{* *} \mathrm{P}<0.01$ and ${ }^{* * * *} \mathrm{P}<0.001$ vs. parental cell line. 5-FU, fluorouracil; EPB, epirubicin; FITC, fluorescein isthiocyanate.

performed using Student's unpaired t-tests. $\mathrm{P}<0.05$ was considered to indicate a statistically significant difference.

\section{Results}

Cells cultured in low-serum medium demonstrate greater sensitivity to fenretinide. The majority of CSC cells are in a quiescent state, which is why the majority of current chemotherapy regimens fail to achieve a cure. To evaluate the effects of fenretinide on quiescent cells, HT29 and HCT116 cells were cultured in serum-starvation conditions, which causes cell cycle arrest at the G0 phase and cell quiescence (Fig. 1A). HT29 and HCT116 cells were incubated separately in low-serum medium and full-serum medium, and their viability was assessed using the MTT cell viability assay following treatment with $6 \mu \mathrm{m}$ fenretinide for 48 or $72 \mathrm{~h}$. Cells incubated in the low-serum culture medium were more sensitive to fenretinide treatment compared with those incubated in full-serum medium (Fig. 1B). In the cell apoptosis assays, marked induction of apoptosis was observed oonly when HCT116 and HT29 cells were treated with fenretinide in low-serum medium (Fig. 1C). These results indicated that the cells grown in low-serum medium demonstrated greater sensitivity to fenretinide.

Colon spheres formed in the serum-free medium demonstrate stem-like characteristics. Previous research has demonstrated that CSCs generate three-dimensional spheres comprised of a small number of CSCs that have the ability to self-renew and generate spheres on serial passage, as well as progenitor cells capable of multi-lineage differentiation (22). Sphere-forming assays are used to enrich CSCs in vitro as an operational surrogate for CSCs. Unsorted HCT116 and HT29 cells form spheres in SFM following 5 days of culture. Spheres with a diameter of 50-100 $\mu \mathrm{m}$ were observed (Fig. 2A).

CSCs are identifiable by their specific surface epitopes. Colon CSCs express the surface markers CD44 and CD133 (23). CD44 has been demonstrated to be a putative marker of colon CSCs in tumor specimens and cell lines (24). Therefore, the expression of CD44 was examined in HT29 parental and sphere cells. The percentage of $\mathrm{CD} 44^{+}$cells was increased in sphere cells compared with parental cells (Fig. 2B).

CSCs are considered to be drivers of tumor initiation and progression. Accordingly, to determine the tumorigenic ability of sphere cells, their ability to grow subcutaneously in immune-deficient mice was analyzed. A total of 10,000 parental cells or sphere cells were injected into the inguinal area of NOD/SCID mice. Significantly increased initiation and growth of tumors were observed only in the inguinal areas injected with the sphere cells (Fig. 2C). This result indicated the potent in vivo self-renewal and tumor-initiating capacities of spheres, which is consistent with the notion that CSCs drive tumor progression.

CSCs demonstrate resistance to a number of conventional therapies, which may explain why it is difficult to eradicate cancer cells completely and why recurrence is an ever-present threat (25). To examine whether sphere cells retained CSC chemo-resistance, the sensitivity of sphere cells to fluorouracil (5-FU, Sigma-Aldrich; Merck KGaA) and epirubicin (EPB, Sigma-Aldrich; Merck KGaA), two first-line chemotherapeutic agents used to treat colon cancer, was assessed. The cell viability of the parental HT29 cells and the sphere cells treated with 5-FU and EPB was assessed. Marked inhibition of cell growth was observed only in the parental HT29 cells treated with fenretinide (Fig. 2D). These results suggested that colon sphere cells mimic the status of colon CSC cells in this setting. 
A
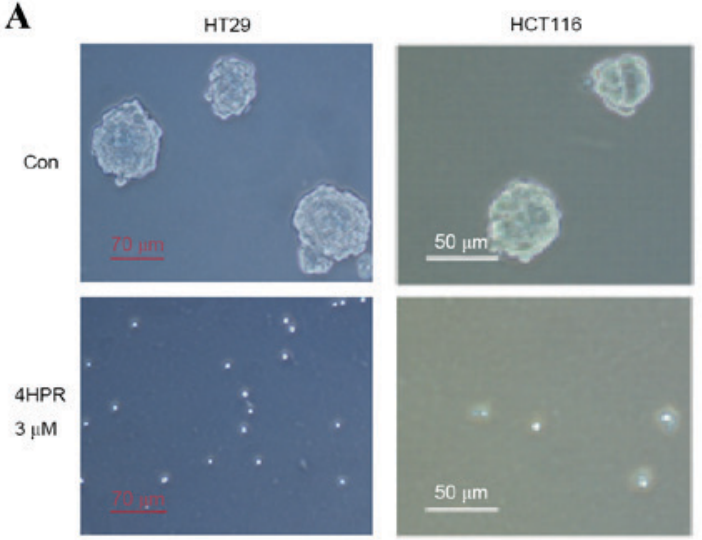

B

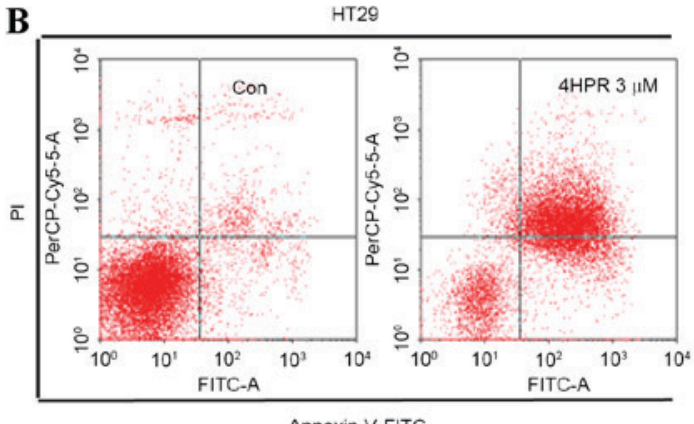

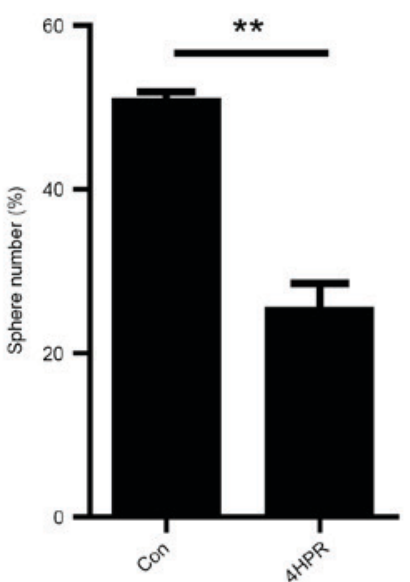

HCT116

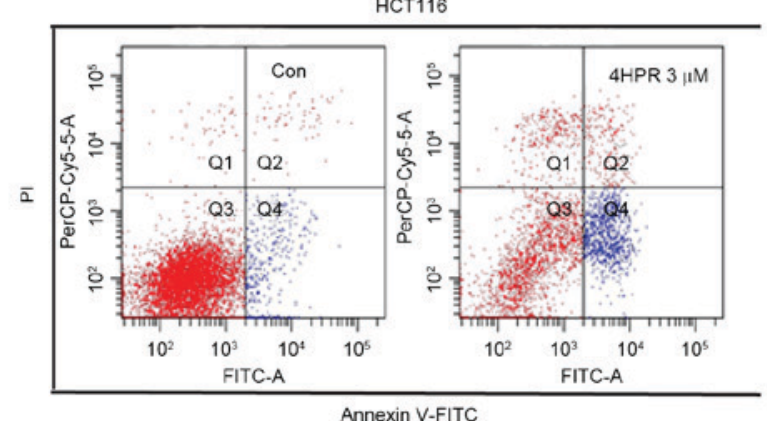

Annexin V-FITC

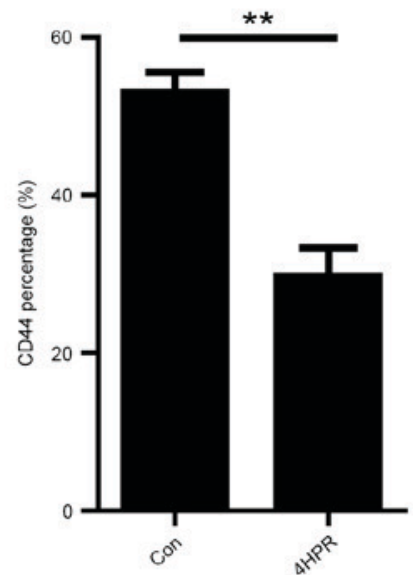

Figure 3. Responses of HT29 and HCT116 sphere cells to 4HPR. (A) HT29 and HCT116 cells were seeded in sphere-forming conditions with and without $3 \mu$ M 4HPR for 5 days, and the spheres were photographed using an inverted microscope (magnification x10). The bar chart indicates the sphere-forming rate of HT29 cells in serum-free medium. (B) Representative dot plots revealing the apoptotic status of HT29 and HCT116 sphere cells. Cells were treated with $3 \mu$ M 4HPR for $48 \mathrm{~h}$. (C) Percentage of CD44+ cells in HT29 cells treated with or without $3 \mu \mathrm{m} 4 \mathrm{HPR}$ for $48 \mathrm{~h}$. Representative dot plots revealing the percentage of $\mathrm{CD} 44^{+}$cells. Data are expressed as the mean \pm standard error of the mean and are representative of at least three independent experiments. ${ }^{* *} \mathrm{P}<0.01$ vs. Con. 4HPR, fenretinide; CD44, cluster of differentiation 44; Con, control; FITC, fluorescein isthiocyanate.

Fenretinide preferentially targets sphere cells. Fenretinide has been demonstrated to preferentially eradicate acute myeloid leukemia (AML) and chronic myeloid leukemia (CML) stem cells, and CSCs in ovarian and breast cancer $(9,10,14)$. Therefore, it is necessary to investigate whether fenretinide can selectively target CSCs in colon cancer and disturb their ability for self-renewal. HT29 and HCT116 parental cells were insensitive to fenretinide (Fig. 1B). However, when cultured under sphere-forming conditions, treatment with $3 \mu \mathrm{m}$ fenretinide inhibited the sphere formation of HT29 and HCT116 cells (Fig. 3A).
Marked induction of apoptosis was observed in HT29 and HCT116 sphere cells following treatment with $3 \mu \mathrm{m}$ fenretinide for $48 \mathrm{~h}$ (Fig. 3B). The percentage of CD $44^{+}$ cells was the measured in HT29 cells treated with or without $3 \mu \mathrm{m}$ fenretinide for $48 \mathrm{~h}$. The percentage of $\mathrm{CD} 44^{+}$cells was lower in the fenretinide-treated cells compared with the corresponding control cells (Fig. 3C). These results suggested that fenretinide is a potential candidate for eradicating colon CSCs, as it reduced the CSC population below the control levels and because the colon sphere cells were more sensitive to fenretinide compared to the cell lines. 
A

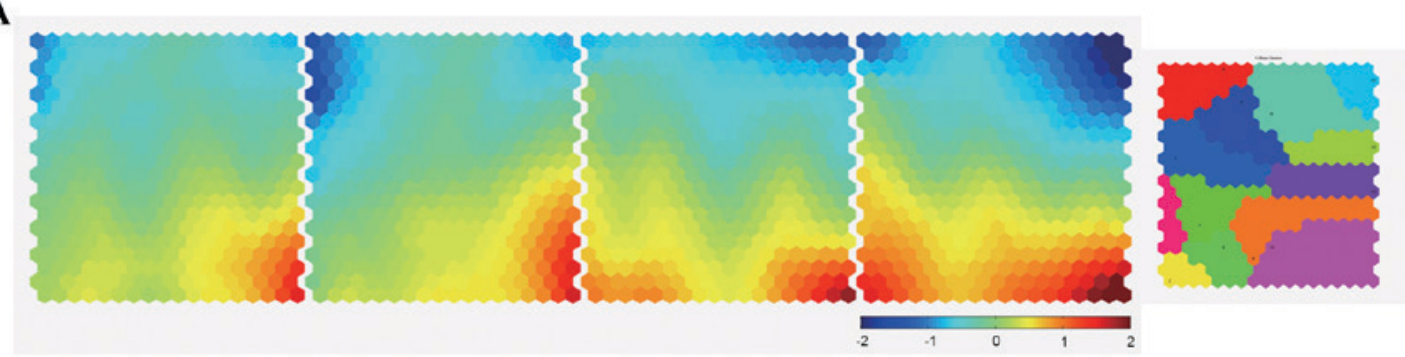

B

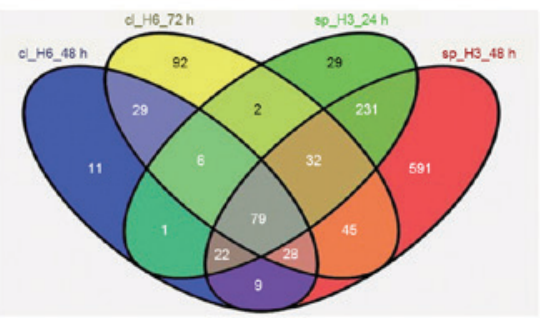

$\mathbf{D}^{\mathrm{so}}$

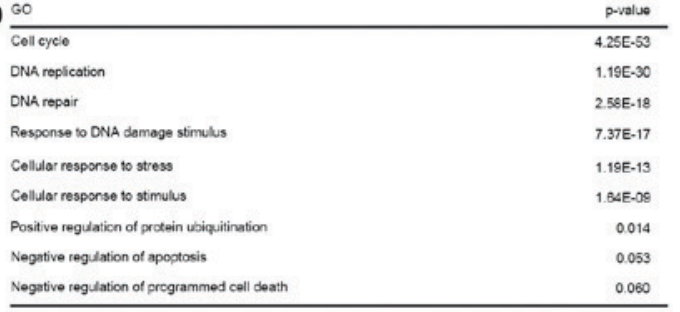

C

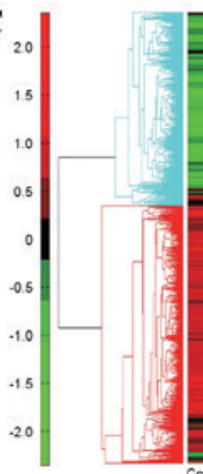

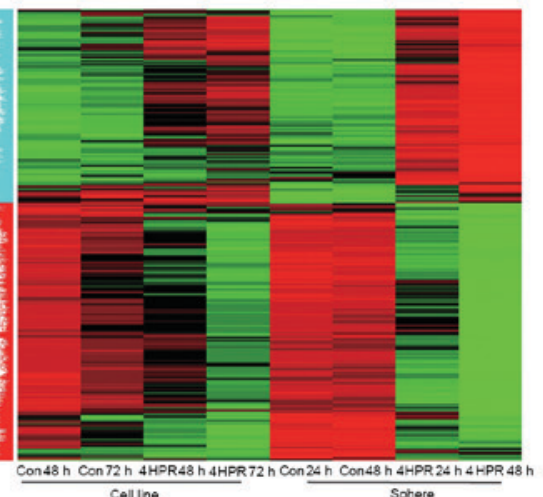

Cell Ine Sphere
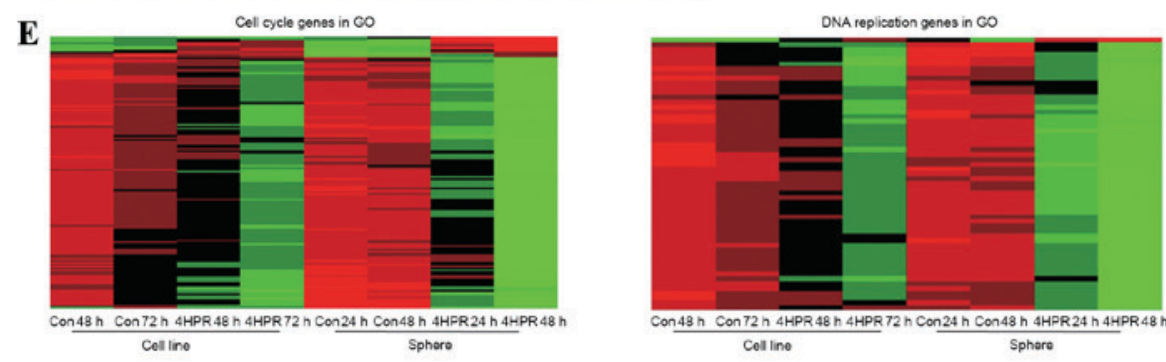

Colv'ar rosponse to stross gones in GO
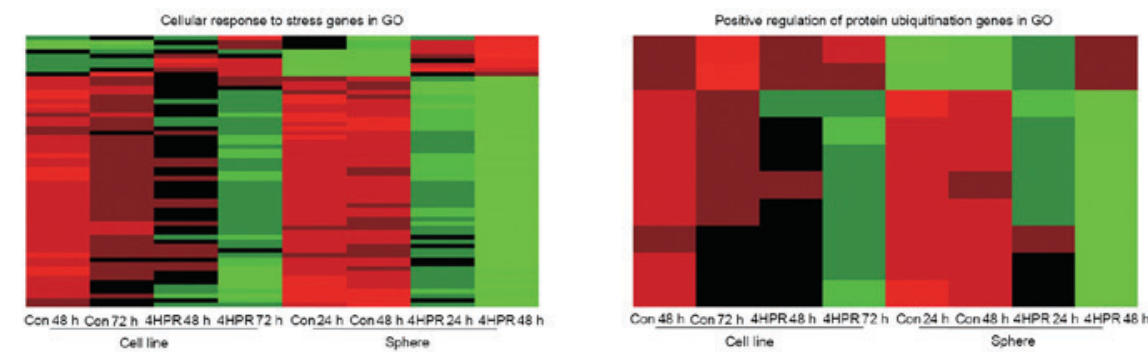

Figure 4. Transcriptome profiles of HT29 enriched sphere cells and parental cells treated with 4HPR. (A) Illustration of transcriptome changes using a Component Plane Presentation, Self-Organizing Map. Each presentation illustrates a sample-specific change, in which the upregulated genes (represented in red), downregulated genes (represented in blue), and moderately regulated genes (represented in yellow and green) are well delineated. The color bar indicates the expression values (log ratio in base 2), and brighter colors indicate higher values. (B) The numbers of regulated genes that were selected based on a 2-fold change threshold in each sample are depicted. (C) Heat map of the microarray data revealing the expression levels of these selected genes. (D) GO and Database for Annotation, Visualization and Integrated Discovery analysis of the selected genes. (E) Heat maps of significant GO clusters, revealing the specific changes in gene regulation. 4HPR, fenretinide; GO, gene oncology; Con, control.

Microarray analysis of HT29 cells treated with fenretinide. To identify the molecular mechanisms underlying fenretinide-induced apoptosis, microarray gene expression profiling of HT29 parental cells and sphere cells was performed. Parental cells were treated with $6 \mu \mathrm{m}$ fenretinide for 48 and $72 \mathrm{~h}$, while sphere cells were treated with $3 \mu \mathrm{m}$ fenretinide for 24 or $48 \mathrm{~h}$, as sphere cells are not able to survive when treated with $\geq 6 \mu \mathrm{m}$ ferentinide or when treated with $3 \mu \mathrm{m}$ for over $72 \mathrm{~h}$. Total mRNA was extracted and profiled using a whole-genome array. To recognize the prominent features in the data, self-organizing map (SOM)-based clustering analysis of the top 5,000 regulated genes was performed. Genes were grouped into 13 clusters, and different changes in expression were observed at each time (Fig. 4A).

The number of target genes in each sample is depicted in Fig. 4B. A total of 851 genes from the sphere samples that did not overlap with the parental cells were then selected for further analysis. These genes exhibited more prominent changes 

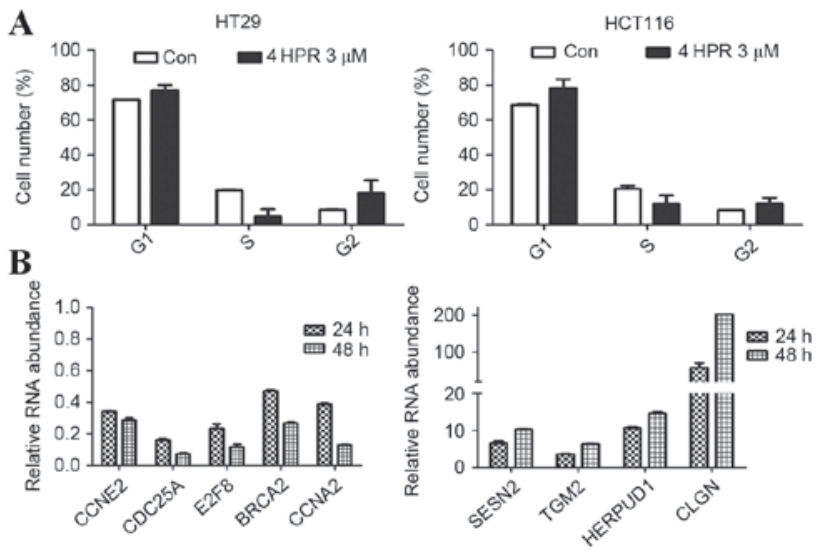

Figure 5. Analysis of the cell cycle, cell cycle-associated genes, and stress response-associated genes. (A) Cell cycle analysis of HCT116 and HT29 sphere cells, assessed using flow cytometry, following treatment with $3 \mu \mathrm{M} 4 \mathrm{HPR}$ for $24 \mathrm{~h}$. The values are expressed as the mean \pm standard deviation $(n=3)$ (B) Reverse transcription-quantitative polymerase chain reaction analysis of the expression of cell cycle-associated genes, and stress response-associated genes. 4HPR, fenretinide; Con, control; CCNE2, cyclin E2; CDC25A, cell division cycle 25A; E2F8, E2F transcription factor 8; BRCA2, BRCA2, DNA repair associated; CCNA2, cyclin A2; SESN2, sestrin 2; TGM2, transglutaminase 2; HERPUD1, homocysteine inducible ER protein with ubiquitin like domain 1; CLGN, calmegin.

following fenretinide treatment, as depicted in Fig. 4C. To reveal the functional relevance of these specifically regulated genes in sphere cells, functional enrichment analysis was performed using DAVID and GO (26). Significant processes associated with specific regulation of sphere cells treated with fenretinide were the cell cycle, DNA replication, cellular response to stress or stimuli, cellular regulation of protein ubiquitination and apoptosis (Fig. 4D). Heat maps of these gene clusters are depicted in Fig. 4E.

Fenretinide induces genes that are associated with cell cycle regulation and stress-responsive activities. Cell cycle-associated genes were the most regulated clusters in the sphere cells. This indicated that the effect of fenretinide treatment on sphere cells was associated with cell cycle regulation. Flow cytometry was used to analyze the cell cycle of HCT116 and HT29 sphere cells. The percentage of sphere cells at the G1 and G2 stages increased and the percentage in cells in S phase decreased following treatment with $3 \mu \mathrm{m}$ fenretinide for $24 \mathrm{~h}(\mathrm{P}<0.05$; Fig. 5A). This result indicated that sphere cell proliferation was inhibited by fenretinide.

Next, certain key genes involved in cell cycle regulation for were selected for RT-qPCR analysis. Compared with genes in the untreated cells, $C C N E 2, C D C 25 A, E 2 F 8, B R C A 2$, and $C C N A 2$ were downregulated following fenretinide treatment (Fig. 5B). CCNE2 encodes cyclin E2, which belongs to the highly-conserved cyclin family. This cyclin forms a complex with and functions as a regulatory subunit of cyclin dependent kinase 2 (CDK2) and is involved in cell cycle G1/S transition. A significantly increased expression level of this gene is observed in tumor-derived cells. CCNA2 encodes cyclin A2, which binds to and activates CDK2 kinases, and thus promotes G1/S and $\mathrm{G} 2 / \mathrm{M}$ cell cycle transitions. CDC25A is a phosphatase that is required for the progression from the $\mathrm{G} 1$ to the $\mathrm{S}$ phase in the cell cycle. It activates the cyclin-dependent kinase CDC2 by removing two phosphate groups. E2F8 regulates the progression from $\mathrm{G} 1$ to $\mathrm{S}$ phase by ensuring the nucleus divides at the proper time. BRCA2 is involved in the maintenance of genome stability, and its mutation confers an increased lifetime risk of developing cancer. These genes are essential for cell cycle control. Fenretinide preferentially downregulated these genes in HT29 sphere cells, which may inhibit cell proliferation and may account for the fenretinide-induced apoptosis.

ROS balance is important for normal cell function, and fenretinide is known to increase cellular ROS levels. Previous research has demonstrated that fenretinide-induced apoptosis is associated with the conversion of oxidative signaling into downstream stress activities, including the redox response, endoplasmic reticulum (ER) stress response/unfolded protein response (UPR), and proteasome activation mediated by certain key stress-responsive regulators (27). The present study used RT-qPCR analysis to analyze certain key genes involved in stress-response regulation. The accumulation of unfolded proteins in the ER triggers the ER stress response. This response includes the inhibition of translation to prevent further accumulation of unfolded proteins, increased expression of proteins involved in polypeptide folding, known as the UPR, and the destruction of misfolded proteins by the ER-associated protein degradation (ERAD) system (28). SESN2 encodes sestrin 2 , which may be involved in cellular responses to different stress conditions. HERPUD1 may be involved in the UPR and ERAD. HEPRUD1 expression is induced by the UPR and has an ER stress response element in its promoter region, whereas the encoded protein has an N-terminal ubiquitin-like domain, which may interact with the ERAD system. This protein has been demonstrated to interact with presenilin proteins and to increase the level of amyloid- $\beta$ protein when overexpressed (29). TGM2 encodes transglutaminase 2 , which is a monomer that is induced by retinoic acid, and may be involved in apoptosis. $C L G N$ encodes a specific ER chaperone protein, calmegin. The observed upregulation of these genes suggested that significant antioxidative activity occurred in sphere cells following fenretinide treatment, and may partially explain why fenretinide preferentially targets sphere cells.

\section{Discussion}

CSCs are widely accepted to be crucial in cancer initiation, propagation, metastasis and relapse (30-32). The residual CSC reservoir is correlated with the prognosis of patients (33). Therefore, CSC-targeted strategies are likely to be effective interventions to increase the responsiveness to traditional therapeutic strategies, and to reduce the risk of local recurrence and metastasis. At present, substantial attention has been focused on the development of strategies to selectively eliminate CSCs in the hope of preventing the recurrence of cancer, and eventually curing the disease $(5,34,35)$.

Fenretinide has emerged as a promising candidate with chemo-preventive properties $(9,10,14)$. Clinical data have provided evidence that fenretinide significantly reduces the risk of secondary breast cancer in premenopausal women and may be able to eliminate cancer cells in the early stages. In vitro studies suggest that the anticancer activity of fenretinide may arise from its ability to induce apoptosis in tumor cells. A number of previous investigations have 
elucidated much about the apoptotic activity of fenretinide. Diverse signaling molecules, including ROS, ceramide and the ganglioside GD3, trigger the activation of cellular stress response pathways and mediate the induction of apoptosis by fenretinide in transformed, premalignant and malignant cells. In the majority of cell types, the apoptotic activity of fenretinide appears to be induced by mechanisms that are independent from retinoic acid receptor activation and ultimately initiate the intrinsic or mitochondria-mediated pathway of cell elimination. Multiple proteins, including various transcription factors and kinases, are responsive to stressful conditions in cells. Once active, these proteins determine cell fate through the initiation of apoptosis. Previous systematic detection of transcriptional changes in response to oxidative signals generated in leukemia cells following fenretinide treatment revealed the appearance of multiple stress-responsive events during fenretinide-induced apoptosis, including the redox response, the ER stress response/UPR, translation repression and proteasome activation (27).

In the present study, colon spheres were demonstrated to form in serum-free medium with stem-like characteristics. The unique anti-CSC effects of fenretinide were stratified in a series of in vitro and in vivo experiments, fenretinide was demonstrated to exert a selective cytotoxic effect on HT29 sphere cells but not on their normal counterparts. Mechanistic studies provided insight into the effects of fenretinide in eradicating colon CSCs. Transcriptome analysis of fenretinide-treated HT29 sphere cells identified several features that highlighted the involvement of cell cycle regulation and activation of ROS-induced stress responses.

ROS are involved in the regulation of CSCs and confers resistance to radiotherapy. The ROS level in CSCs is low, which makes it difficult to eliminate them by traditional radiotherapy (36). However, the low ROS levels in CSCs provide an opportunity to preferentially target CSC cells using ROS inducers, including fenretinide. Stress-responsive activities, including elevation of ROS levels in cells treated with fenretinide, may explain the selective effect on colon stem-like cells. However, further studies are required to improve understanding of the specific underlying mechanisms.

Fenretinide is a promising agent that is able to selectively target CSCs. Fenretinide does not induce point mutations or chromosomal aberrations and causes few adverse side effects, suggesting that fenretinide may be compatible for long-term use as a chemo-preventive agent (37). Further investigations are warranted to gain improved insight into its specific functions and the underlying mechanisms.

To the best of our knowledge, the present study was the first to investigate the effect of fenretinide on colon stem cells. Fenretinide is a promising agent that is able to selectively target CSCs. Fenretinide preferentially targeted colon sphere cells, which are believed to possess certain stem-like characteristics. These results are an important addition to current knowledge about fenretinide, and provide a foundation for its clinical application in the treatment of cancer.

\section{Acknowledgements}

The authors would like to thank Professor Zi-Jiang Chen for her helpful suggestion and assistance.

\section{Funding}

The present study was supported in part by grants from the National Natural Science Foundation (grant no. 81170503) and Ministry of Science and Technology of China (grant no. 2013CB966802).

\section{Availability of data and materials}

The datasets used and analyzed during the current study are available from the corresponding author on reasonable request. And the microarray data are available at the GEO accession no. GSE66983.

\section{Author's contributions}

LL and YD designed the present study. JL and HW performed the animal experiments. LL performed the experiments. LL, JL, HZ and YD analyzed the data. LL and YD wrote the article. JL and YD provided final approval of the version to be published.

\section{Ethics approval and consent to participate}

The animal experiments were approved by the Committee on Laboratory Animal Research of Shanghai Jiao Tong University (Shanghai, China), and were conducted according to the guidelines of the Laboratory Animal Center of Shanghai Jiao Tong University School of Medicine (Shanghai, China).

\section{Consent for publication}

The present study did not use patient information and related samples.

\section{Competing interests}

The authors declare that they have no competing interests.

\section{References}

1. Siegel R, Naishadham D and Jemal A: Cancer statistics, 2013. CA Cancer J Clin 63: 11-30, 2013.

2. Scopelliti A, Cammareri P, Catalano V, Saladino V, Todaro M and Stassi G: Therapeutic implications of cancer initiating cells. Expert Opin Biol Ther 9: 1005-1016, 2009.

3. Ricci-Vitiani L, Lombardi DG, Pilozzi E, Biffoni M, Todaro M, Peschle C and De Maria R: Identification and expansion of human colon-cancer-initiating cells. Nature 445: 111-115, 2007.

4. Hanahan D and Weinberg RA: Hallmarks of cancer: The next generation. Cell 144: 646-674, 2011.

5. Todaro M,Francipane MG, Medema JP and Stassi G: Colon cancer stem cells: Promise of targeted therapy. Gastroenterology 138: 2151-2162, 2010.

6. Moon RC, McCormick DL, Becci PJ, Shealy YF, Frickel F, Paust $J$ and Sporn MB: Influence of 15 retinoic acid amides on urinary bladder carcinogenesis in the mouse. Carcinogenesis 3: 1469-1472, 1982

7. Ohshima M, Ward JM and Wenk ML: Preventive and enhancing effects of retinoids on the development of naturally occurring tumors of skin, prostate gland, and endocrine pancreas in aged male ACI/segHapBR rats. J Natl Cancer Inst 74: 517-524, 1985.

8. Malone W, Perloff M, Crowell J, Sigman C and Higley H: Fenretinide: A prototype cancer prevention drug. Expert Opin Investig Drugs 12: 1829-1842, 2003. 
9. Du Y, Xia Y, Pan X, Chen Z, Wang A, Wang K, Li J and Zhang J: Fenretinide targets chronic myeloid leukemia stem/progenitor cells by regulation of redox signaling. Antioxid Redox Signal 20: 1866-1880, 2014.

10. Zhang H, Mi JQ, Fang H, Wang Z, Wang C, Wu L, Zhang B, Minden M, Yang WT, Wang HW, et al: Preferential eradication of acute myelogenous leukemia stem cells by fenretinide. Proc Natl Acad Sci USA 110: 5606-5611, 2013.

11. Lovat PE, Ranalli M, Bernassola F, Tilby M, Malcolm AJ, Pearson AD, Piacentini M, Melino G and Redfern CP: Distinct properties of fenretinide and CD437 lead to synergistic responses with chemotherapeutic reagents. Med Pediatr Oncol 35: 663-668, 2000.

12. Maurer BJ, Melton L, Billups C, Cabot MC and Reynolds CP: Synergistic cytotoxicity in solid tumor cell lines between $\mathrm{N}$-(4-hydroxyphenyl)retinamide and modulators of ceramide metabolism. J Natl Cancer Inst 92: 1897-1909, 2000.

13. Shan D, Gopal AK and Press OW: Synergistic effects of the fenretinide (4-HPR) and anti-CD20 monoclonal antibodies on apoptosis induction of malignant human B cells. Clin Cancer Res 7: 2490-2495, 2001

14. Wang H, Zhang Y and Du Y: Ovarian and breast cancer spheres are similar in transcriptomic features and sensitive to fenretinide. Biomed Res Int 2013: 510905, 2013.

15. Livak KJ and Schmittgen TD: Analysis of relative gene expression data using real-time quantitative PCR and the 2(-Delta Delta C(T)) method. Methods 25: 402-408, 2001.

16. Lockhart DJ, Dong H, Byrne MC, Follettie MT, Gallo MV, Chee MS, Mittmann M, Wang C, Kobayashi M, Horton H and Brown EL: Expression monitoring by hybridization to high-density oligonucleotide arrays. Nat Biotechnol 14: 1675-1680, 1996

17. Dennis G Jr, Sherman BT, Hosack DA, Yang J, Gao W, Lane HC and Lempicki RA: DAVID: Database for annotation, visualization, and integrated discovery. Genome Biol 4: P3, 2003.

18. Gene Ontology Consortium: Gene ontology consortium: Going forward. Nucleic Acids Res 43 (Database Issue): D1049-D1056, 2015.

19. Edgar R, Domrachev M and Lash AE: Gene expression omnibus: NCBI gene expression and hybridization array data repository. Nucleic Acids Res 30: 207-210, 2002.

20. Du Y, Wang K, Fang H, Li J, Xiao D, Zheng P, Chen Y, Fan H, Pan X, Zhao C, et al: Coordination of intrinsic, extrinsic, and endoplasmic reticulum-mediated apoptosis by imatinib mesylate combined with arsenic trioxide in chronic myeloid leukemia. Blood 107: 1582-1590, 2006.

21. Xiao L, Wang K, Teng Y and Zhang J: Component plane presentation integrated self-organizing map for microarray data analysis. FEBS Lett 538: 117-124, 2003.

22. Dontu G, Abdallah WM, Foley JM, Jackson KW, Clarke MF, Kawamura MJ and Wicha MS: In vitro propagation and transcriptional profiling of human mammary stem/progenitor cells Genes Dev 17: 1253-1270, 2003.
23. Haraguchi N, Ohkuma M, Sakashita H, Matsuzaki S, Tanaka F, Mimori K, Kamohara Y, Inoue H and Mori M: CD133+CD44+ population efficiently enriches colon cancer initiating cells. Ann Surg Oncol 15: 2927-2933, 2008

24. Du L, Wang H, He L, Zhang J, Ni B, Wang X, Jin H, Cahuzac N, Mehrpour M, Lu Y and Chen Q: CD44 is of functional importance for colorectal cancer stem cells. Clin Cancer Res 14: 6751-6760, 2008.

25. Park CY, Tseng D and Weissman IL: Cancer stem cell-directed therapies: Recent data from the laboratory and clinic. Mol Ther 17: 219-230, 2009.

26. Gene Ontology Consortium: The gene ontology project in 2008. Nucleic Acids Res 36 (Database Issue): D440-D444, 2008.

27. Wang K, Fang H, Xiao D, Zhu X, He M, Pan X, Shi J, Zhang H, Jia X, Du Y and Zhang J: Converting redox signaling to apoptotic activities by stress-responsive regulators HSF1 and NRF2 in fenretinide treated cancer cells. PLoS One 4: e7538, 2009.

28. Szegezdi E, Logue SE, Gorman AM and Samali A: Mediators of endoplasmic reticulum stress-induced apoptosis. EMBO Rep 7: 880-885, 2006

29. Li X, Uemura K, Hashimoto T, Nasser-Ghodsi N, Arimon M, Lill CM, Palazzolo I, Krainc D, Hyman BT and Berezovska O: Neuronal activity and secreted amyloid $\beta$ lead to altered amyloid $\beta$ precursor protein and presenilin 1 interactions. Neurobiol Dis 50: 127-134, 2013.

30. Ward RJ and Dirks PB: Cancer stem cells: At the headwaters of tumor development. Annu Rev Pathol 2: 175-189, 2007.

31. Tang DG: Understanding cancer stem cell heterogeneity and plasticity. Cell Res 22: 457-472, 2012.

32. Kleffel S and Schatton T: Tumor dormancy and cancer stem cells: Two sides of the same coin? Adv Exp Med Biol 734: 145-179, 2013.

33. Shien K, Toyooka S, Ichimura K, Soh J, Furukawa M, Maki Y, Muraoka T, Tanaka N, Ueno T, Asano $\mathrm{H}$, et al: Prognostic impact of cancer stem cell-related markers in non-small cell lung cancer patients treated with induction chemoradiotherapy. Lung Cancer 77: 162-167, 2012.

34. Dean M, Fojo T and Bates S: Tumour stem cells and drug resistance. Nat Rev Cancer 5: 275-284, 2005.

35. Jordan CT, Guzman ML and Noble M: Cancer stem cells. N Engl J Med 355: 1253-1261, 2006.

36. Diehn M, Cho RW, Lobo NA, Kalisky T, Dorie MJ, Kulp AN, Qian D, Lam JS, Ailles LE, Wong M, et al: Association of reactive oxygen species levels and radioresistance in cancer stem cells. Nature 458: 780-783, 2009.

37. Hail NJr,Kim HJ and Lotan R: Mechanisms of fenretinide-induced apoptosis. Apoptosis 11: 1677-1694, 2006. 